\title{
Bounds on the Solar Antineutrino total Flux and Energy spectrum from the SK experiment
}

\author{
E. Torrente-Lujan. \\ IFIC-Dpto. Fisica Teorica. CSIC-Universitat de Valencia. Dr. Moliner 50, E-46100, Burjassot, Valencia, Spain. \\ e-mail: e.torrente@cern.ch
}

\begin{abstract}
A search for inverse beta decay electron antineutrinos has been carried out using the 825 days sample of solar data obtained at SK. The absence of a significant signal, that is, contributions to the total SK background and their angular variations has set upper bounds on a) the absolute flux of solar antineutrinos originated from ${ }^{8} B$ neutrinos $\Phi_{\bar{\nu}}\left({ }^{8} B\right)=<1.8 \times 10^{5} \mathrm{~cm}^{-2} \mathrm{~s}^{-1}$ which is equivalent to an averaged conversion probability bound of $P<3.5 \%$ (SSM-BP98 model) and b) their differential energy spectrum, the conversion probability is smaller than $8 \%$ for all $E_{e, v i s}>6.5$ $\mathrm{MeV}$ going down the $5 \%$ level above $E_{e, v i s} \approx 10 \mathrm{MeV}$. It is shown that an antineutrino flux would have the net effect of enhancing the SK signal at hep neutrino energies. The magnitude of this enhancement would highly depend on the, otherwise rather uncertain at this moment, steepness of the solar neutrino spectrum at these energies.
\end{abstract}

Introduction. The combined action of spin flavor precession in a magnetic field and ordinary neutrino matter oscillations can produce an observable flux of $\bar{\nu}_{e R}$ 's from the Sun in the case of the neutrino being a Majorana particle. In the simplest model, where a thin layer of highly chaotic of magnetic field is assumed at the bottom of the convective zone $\left(R \sim 0.7 R_{\odot}\right)$, the antineutrino appearance probability at the exit of the layer can

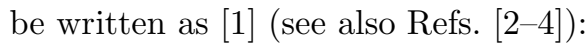

$$
P\left(\nu_{e L} \rightarrow \tilde{\nu}_{e R}\right)_{f}=\xi P\left(\nu_{e L} \rightarrow \nu_{\mu L}\right)_{i},
$$

where the parameter $1-2 \xi \sim \exp \left(-4 \Omega^{2} \Delta r\right)$ includes the layer width $\left(\Delta r \sim 0.1 R_{\odot}\right)$ and the r.m.s strength of the field. The antineutrino flux could be large if, i.e., the neutrino have passed through a MSW resonance before arriving to the layer. The MSW resonance converts practically all the initial $\bar{\nu}_{e}$ flux into $\bar{\nu}_{\mu}$. The field finally converts them into $\bar{\nu}_{e}$. A fraction of the $\bar{\nu}_{e}$ will be reconverted into $\bar{\nu}_{\mu}$ by mass oscillations but this reconversion is limited in this case by the chaotic character of the process.

Water Cerenkov detectors such as Kamiokande and SuperKamiokande (SK) which are sensitive to the $\nu_{e}-e$ elastic interaction are also capable of detecting these $\bar{\nu}_{e}$ 's coming from the sun. Forthcoming solar neutrino experiments, such as SNO and Borexino are also expected to have a high sensitivity to them. The specific signature of electron antineutrinos in proton containing materials is the inverse beta decay process: $\bar{\nu}_{e}+p \rightarrow$ $n+e^{+}$, which produces almost isotropical monoenergetic positrons with a relatively high cross section. Antineutrino events would contribute to the background to forward-peaked neutrino solar events.

The residual angular correlation between the direction of the incident neutrino and the resulting electron was proposed already in Ref. [5] as a way of detecting solar neutrinos. More recently, it was again suggested [6] the same procedure to separate statistically antineutrino events in the SK sample. In practice however, as it was pointed out in Ref. [9] and we show in detail in this work, the angular distribution is of a more complicated nature than was naively assumed in Ref. [6]. Nevertheless, as we show in the detailed analysis presented here, meaningful bounds on solar antineutrino fluxes and appearance probability can be obtained from the SK data.

Solar antineutrinos could have been already detected at SK: due to the anomalous, forward-peaked at the higher energies, angle distribution the excess of high energy solar neutrino events observed in SK above $13 \mathrm{MeV}$, the hep spectrum anomaly [7,8], could be explained, albeit partially, as an excess of positrons coming from inverse beta decay appearing with a direction which fakes that one of real solar neutrino electrons.

Antineutrino Cross sections. In the limit of infinite nucleon mass, the cross section for the reaction $\bar{\nu}_{e}+p \rightarrow n+e^{+}$is given by [10, 5$] \sigma\left(E_{\bar{\nu}}\right)=c_{1} E_{e} p_{e}$. The transition matrix element $c_{1}=2 \pi^{2} \log 2 / m_{e}^{5} f t_{1 / 2}$ is expressed in terms of the free neutron decay $f t_{1 / 2}$ value, where the phase-space factor $f=1.71465 \pm 0.00015$ follows from calculation [11] and $t_{1 / 2}=614.6 \pm 1.3 \mathrm{sec}$ is latest published value for the neutron half-life [12]. With $M_{n}, M_{p}$ and $m_{e}$ being the masses of neutron, proton and electron, respectively and $\Delta M=M_{n}-M_{p} \simeq 1.293 \mathrm{MeV}$.

The energy $E_{\bar{\nu}}$ of the incident neutrino is related to that one of the positron $E_{e^{+}}$by: $E_{\bar{\nu}}^{0}=E_{e^{+}}+\Delta M$. From the values above, we obtain: $c_{1}=(9.54 \pm 0.02) \times 10^{-44}$ $\mathrm{cm}^{2} / \mathrm{MeV}^{2}$. Corrections to the total cross-section due to weak magnetism arising from the difference in the anomalous magnetic moments of the neutron and proton and radiative corrections including internal bremsstrahlung (see Ref. [10] and references therein) are very small and can be neglected for our purposes.

Direct recoil corrections to the total cross section can also be neglected in principle. However, recoil corrections are potentially important in relating the positron and antineutrino energies in order to evaluate the antineutrino flux. The positron spectrum is not monoenergetic in this case and one has to integrate over the positron 
angular distribution to obtain the positron yield. At the SK experiment, because the antineutrino flux $\Phi\left(E_{\nu}\right)$ would typically decrease quite rapidly with energy, the lack of adequate corrections will systematically overestimate the positron yield. For the SK case and taking into account the SSM-BP $98^{8} B$ spectrum, the effect decrease the positron yield by $2-8 \%$ at the main visible energy range $\sim 6-10 \mathrm{MeV}$. The positron yield could decrease up 50\% at hep neutrino energies, a region where incertitudes in the total and differential spectrum are of comparable size or larger. Finite energy resolution smearing will however diminish this correction when integrating over large enough energy bins: in the range $6.5-20 \mathrm{MeV}$ the net positron suppression is estimated to be at the $5 \%$ level, increasing up $20 \%$ at hep energies.

Indirect recoil effects through the antineutrino flux spectrum are also present in the angular distribution as we discuss below.

The angular distribution. For low antineutrino energies, the positron angular distribution is well-described by the expression

$$
\frac{d \sigma}{d \cos \theta}=\sigma\left(E_{\nu}\right) \frac{1}{2}\left(1-v_{e} \alpha\left(E_{\nu}\right) \cos \theta\right)
$$

where $\theta$ is the lab angle between the antineutrino and positron directions, $v_{e}$ is the positron velocity. Except near threshold, this velocity is nearly unity and can be ignored. For a linear distribution as that given by Eq.(2), the values for the average cosine and the $\alpha$ coefficient are related by: $\alpha=3\langle\cos \theta\rangle / v_{e}$. In the limit of infinite nucleon mass, the coefficient $\alpha=\alpha^{(0)} \equiv\left(\eta^{2}-1\right) /\left(3 \eta^{2}+1\right) \approx$ 0.10 is independent of $E_{\nu}, \eta \equiv g_{A} / g_{V}$ is the ratio between axial and vector couplings of the neutron. Thus the angular distribution of the positrons is weakly backwards, independent of the energy above the threshold region.

The situation greatly changes when weak magnetism and $O(1 / M)$ recoil corrections are kept as is shown in Ref. [9]. At higher energies, terms proportional to higher powers of $\cos \theta$ appear. The average cosine is changed by a noticeable amount. It changes sign for not-so-high energies $\left(E_{\nu} \sim 12-13 \mathrm{MeV}\right)$, the distribution becomes then forward peaked. At first order $O(1 / M)$, we have $\left(E_{\nu}>\approx 5 \mathrm{MeV}\right)$

$$
\frac{d \sigma^{(1)}}{d \cos \theta}=\frac{\sigma(E)}{2}\left(1-\alpha^{(1)} \cos \theta+\beta \cos ^{2} \theta\right)
$$

where the coefficients $\alpha^{(1)}, \beta$ can be read off from Eq.(19) in Ref. [9]. The average cosine can be computed from here yielding the following expression which is an accurate approximation valid from the threshold up $E_{\nu} \approx 100$ $\operatorname{MeV}\left(f_{2}=\mu_{p}-\mu_{n}=3.706\right.$ is the anomalous nucleon magnetic moment, $\left.\eta^{\prime}=4 \eta / 3(1-\eta)\right)$ [9]:

$$
\begin{aligned}
\langle\cos \theta\rangle^{(1)} & =v_{e} \alpha^{0} / 3+\left(1+\left(1+f_{2}\right) \eta^{\prime} a^{0}\right) E_{\nu} / M \\
& \simeq-0.034 v_{e}+2.55 \times 10^{-3} E_{\nu}(M e V) .
\end{aligned}
$$

The angular differential cross section (3) is shown in Fig.(2) for different neutrino energies. It is important to notice that the angular distribution is still linear to a high degree. We can rewrite an effective expression which is a very good approximation to the first order expression in all the energy range. It can be shown, by obvious arguments or, if prefered, by a rigorous Least Square approach, that the best linear fit to the quadratic expression (3) is given by

$$
\frac{d \sigma^{(1, l i n)}}{d \cos \theta}=\frac{\sigma(E)}{2}\left(1-\alpha_{e f f}^{(1)}(E) \cos \theta\right) .
$$

where the effective constant is:

$$
\alpha_{e f f}^{(1)}\left(E_{\nu}\right)=3\langle\cos \theta\rangle^{(1)}
$$

and $\langle\cos \theta\rangle^{(1)}$ is given by expression (14). The angular distribution can be completely parametrized by a single quantity which is a linear function of the neutrino energy.

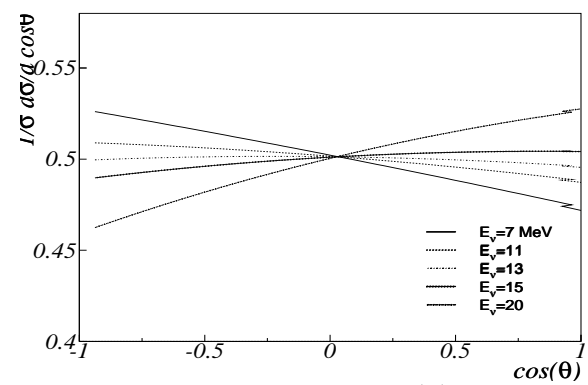

FIG. 1. The angular distribution, Eq.(3), for different antineutrino energies. Above $E_{\nu} \sim 13 \mathrm{MeV}$ the distribution is peaked forwards.

Recoil corrections to the angle positron distribution are important when relating positron and antineutrino energies. In fact they become the dominant effect at the highest SK energies. The observable quantity is the positron angular distribution as a function of the positron energy itself $d N_{e} / d \cos \theta\left(E_{e}\right) \sim d \sigma / d \cos \theta\left(E_{e}\right) \times \Phi_{\nu}\left[E_{\nu}(\cos \theta)\right]$. with $E_{\nu}(\cos \theta)=E_{\nu}^{0} /\left(1-E_{e^{+}}(1-\cos \theta) / M\right)$. At the same positron energy, the difference of the energy of the parent antineutrino for positrons respectively emitted at backward and forward energies is of order $O(1 / M)$ but increasing quadratically with the positron energy:

$$
\Delta E_{\bar{\nu}} \simeq-2 E_{e}\left(E_{e}+\Delta M\right) / M
$$

at $E_{e} \approx 12-15 \mathrm{MeV}$, the difference $\Delta E_{\bar{\nu}} \approx 0.50 \mathrm{MeV}$. The signal difference seen between the forward and backward directions $A \equiv \frac{F-B}{F+B}$ is related to the average cosine. For our angle distribution, we have $A=(3 / 2)\langle\cos \theta\rangle=$ $\alpha / 2$. We have, schematically,

$$
\alpha_{N} \propto \Delta N_{e^{+}} \sim \int d E_{e}\left|\frac{\partial \Phi}{\partial E_{\nu}}\right| \Delta E_{\bar{\nu}} .
$$


It is estimated that, for $1 \mathrm{MeV}$ positron energy bins at $E_{e} \sim 13 \mathrm{MeV}$, the effective angular positron distribution is forward peaked with $\alpha_{N} \sim 0.4-0.5$ (to be compared with values in Fig.(1).

Antineutrino asymmetry and high energy neutrino enhancement. In the SuperKamiokande experiment, the extraction of the solar neutrino signals from the final sample is basically carried out [13] using directional correlation of the events to the direction of the sun, since the recoil electrons keep the directionality of the incoming neutrino. The distribution of the angle between the reconstructed direction and the direction of the sun presents a peak in the sun direction, $\cos \theta=1$. The flat component for $\cos \theta<\approx 0.4$ is considered as background. the excess above this baseline is defined as the solar neutrino signal.

In order to count number of the solar neutrino events, a maximum likelihood method is employed. Signal and background probability functions $\left(P_{s}, P_{b g}\right)$ are defined which depend on angles and energies, due to the finite, energy dependent, angular resolution of the detector. The background probability density is obtained from the data. In the ideal case, the function does not depend on $\cos \theta$ and $P_{b g}=1 / 2$. In the real case, backgrounds, such as $\gamma$-rays, which may have strong directionality in the detector coordinate system are eliminated by fitting a multi-order, energy dependent, polynomial to the detector coordinate distribution and and mapping it back to the direction of the sun. Clearly, this kind of methods does not eliminate a systematic forwardpeaked background as antineutrinos which is virtually un-distinguishable from the real neutrinos. The net effect being that the real signal is systematically underestimated at low energies and, to a much higher degree, overestimated at higher visible energies. As it is implied by the results to be presented below where we use the standard model neutrino spectrum, the net effect is too small in practice to explain the experimental excess [7.8. It is intriguing anyway that the vanishing and sign change of the antineutrino asymmetry occurs precisely at right position of the high energy neutrino spectrum $E_{\nu} \approx 12.5 \mathrm{MeV}$. Irrespective of the total flux, the situation could change if the spectrum profile, through the quantity $\left|\partial \Phi / \partial E_{\nu}\right|$, highly deviates from the assumed standard value.

Experimental angular distributions. The direction of the positron from antineutrino capture is smeared away by the angular resolution of the detector. This angular resolution is energy dependent and due to it, the effective, experimentally detectable, asymmetry parameter $\alpha_{e f f}$ is slightly smaller than the theoretical one and includes an additional small energy dependence.

We have performed a simple Monte Carlo simulation in order to compute the influence of such effect in the SK data. We have found convenient to parameterize the SK angular resolution in terms of the Beta distribution $\epsilon(\theta)_{m, n} \sim \theta^{m-1}(1-\theta)^{n-1}$, the parameters $m, n$ has been obtained from a fit to the data presented in Ref. [13,14]. In particular the variance corresponding to the Beta distribution can directly be obtained from a fit to the energy dependent angular resolution presented in Ref. [13]. Full concrete expressions for $\sigma_{E}$ will be presented in Ref. [15]. We have found that the energy dependence of $\alpha_{e f f}$ can be parametrized as a simple linear expression.

The main conclusion is that the net effect of the finite angular resolution is the smearing of the initial angular distribution consistent with a reduction of no more $15 \%$ on $\alpha_{\text {eff }}$ at the lowest energies observable at SK. At the highest energies, the effect of the detection angular resolution decreases becoming negligible.

Expected antineutrino flux. The average number of positrons $N_{i}$ which are detected per visible energy bin $\Delta E_{i}$ is given by the convolution of different quantities

$$
\begin{aligned}
& N_{i}=Q_{0} \int_{\Delta E_{i}} d E_{e} \int_{0}^{\infty} d E_{e}^{r} \epsilon\left(E_{e}\right) f\left(E_{e}, E_{e}^{r}\right) \\
& \times \int_{E_{e}^{r}}^{\infty} d E_{\bar{\nu}} \bar{F}\left(E_{\bar{\nu}}\right) \sigma\left(E_{\bar{\nu}}, E_{e}^{r}\right)
\end{aligned}
$$

where $Q_{0}$ is a normalization constant accounting for the fiducial volume and live time, $\bar{F}\left(E_{\bar{\nu}}\right)$ is the flux of solar antineutrinos per unit energy at the detector. When ignoring recoil effects the antineutrino capture cross section $\sigma\left(E_{\bar{\nu}}, E_{e}^{r}\right)$ is simply given by $\sigma\left(E_{\bar{\nu}}, E_{e}^{r}\right)=\sigma\left(E_{\bar{\nu}}\right) \delta\left(E_{\bar{\nu}}-\right.$ $\left.E_{e}^{r}\right)$ with $\sigma\left(E_{\bar{\nu}}\right)$ given as before. The functions $\epsilon\left(E_{e}\right)$ and $f\left(E_{e}, E_{e}^{r}\right)$ are, respectively, the detection efficiency and the energy resolution function which includes trigger and VD efficiencies. Expressions for the functions $\epsilon, f$ has been obtained by us using the data presented in Refs. 13,14. Further analysis and explicit expressions can be found in Ref. [15]. The antineutrino flux, production probability $P_{\bar{\nu}_{e}}\left(E_{\nu}\right)$ and the solar-originated neutrino flux $F$ are related by $\bar{F}=F \times P_{\bar{\nu}_{e}}\left(E_{\nu}\right)$.

Results. We have analyzed the data corresponding to the full energy range $6.5-20 \mathrm{MeV}$ from the first 504 days of operation of SK as reported in Ref. [13] as well as the preliminary LE-trigger data corresponding to the first 703 and 825 days of operation 16,17 . In addition we have analyzed data corresponding to individual energy bins of $0.5 \mathrm{MeV}$ interval as presented in Ref. [13]. Note that, in the high energy end, individual energy bins $14-$ 15, $15-16$ and $16-20 \mathrm{MeV}$ are also considered.

Limits from integrated background data. The results obtained from the analysis of the angle-integrated background data over the full energy range are summarized in Table (II) (see second column). From the condition $N_{i}<B a c k_{e x p}$, a model independent upper limit on the flux of antineutrinos originated from ${ }^{8} B$ neutrinos is obtained $\Phi_{\bar{\nu}}\left({ }^{8} B\right)<7.5 \times 10^{5} \mathrm{~cm}^{-2} \mathrm{~s}^{-1}$. Note that this limit would include antineutrinos with energies covering all the ${ }^{8} B$ energy range. This number is equivalent to an upper bound $P<14 \%$ CI95\% on the energy averaged neutrino- 
antineutrino conversion probability (SSM-BP98). Note that much more strict bounds will be obtained below.

The corresponding results obtained from the analysis of individual energy bins are shown in the Figs.(2). In Fig.(2) (top) we show per each positron visible energy bin (width $\pm 0.25 \mathrm{MeV}$, except higher energies) the observed flat background, the maximal SSM (BP98, see Ref. 18]) expected positron signal (supposing $P=1$ ). In the bottom figure (solid circles) we present upper limits on $P$ which are inferred. We observe that the average conversion probability is below $8-10 \%$ for energies above $9 \mathrm{MeV}$ and below the $5 \%$ line for visible energies above $10 \mathrm{MeV}$. In Fig.(3) we present the same information in a slightly different, smoother way, for integrated bins above a certain threshold: the solid circles represent upper limits on $\mathrm{P}$ obtained from the consideration of the global background.

The limits from global background are complemented, specially in the lower energies, with those obtained from consideration of the their angle distribution as follows.

\begin{tabular}{|c|c|c|}
\hline \hline Live Time & Back $k_{\exp }$ & $\left.\Phi_{\bar{\nu}}{ }^{8} B\right)$ \\
\hline 504 days & $7.05 \pm 0.03$ & $<7.7 \times 10^{5}$ \\
703 days & $6.98 \pm 0.03$ & $<7.6 \times 10^{5}$ \\
825 days & $6.87 \pm 0.02$ & $<7.5 \times 10^{5}$ \\
\hline \hline
\end{tabular}

TABLE I. SK live time data. $B a c k_{E x p}(\mathrm{evt} / \mathrm{kt} /$ day $)$ : observed flat background. Limits on the antineutrino flux $\Phi_{\bar{\nu}}\left({ }^{8} B\right)\left(\mathrm{cm}^{-2} \mathrm{~s}^{-1}\right)$. We have supposed for the antineutrino a solar neutrino spectrum with a ${ }^{8} \mathrm{~B}$ absolute flux $\Phi_{\nu}\left({ }^{8} B\right)=5.15_{-0.72}^{+0.98} \times 10^{6} \mathrm{~cm}^{-2} \mathrm{~s}^{-1} 18$. This absolute flux would yield a maximal quantity of $48.2 \mathrm{evt} / \mathrm{kt} /$ day antineutrinos observable in SK.
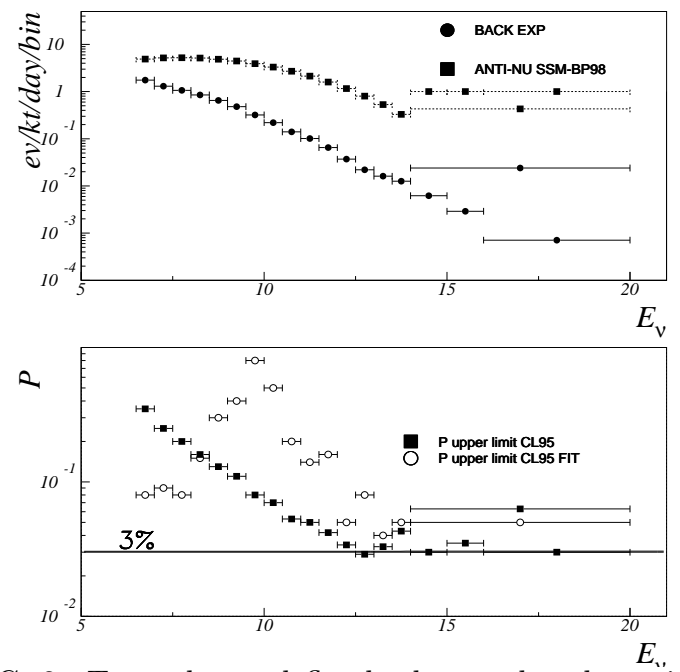

FIG. 2. Top: observed flat background and maximal expected positron signal. Bottom: upper limits on antineutrino conversion probability obtained from global counting (solid circles) and angle fit (empty circles).

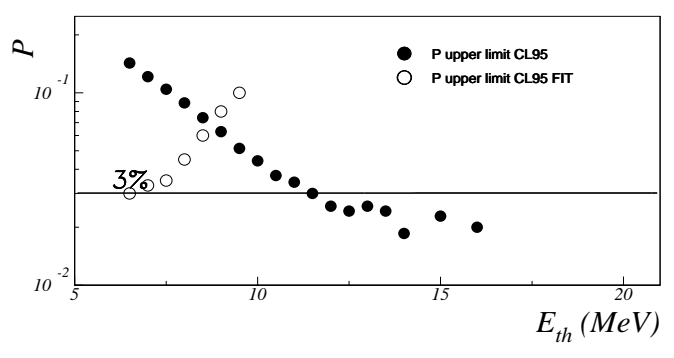

FIG. 3. Upper limits on antineutrino conversion probability as a function of $E_{e^{+}, t h}^{v i s}$. As Fig.(伍)

Limits from fit to the angular distribution. Bounds on the antineutrino flux have been extracted from the fit to the counting yield in the angular region where "background" events from $\nu e$ interactions can be neglected. For this purpose the use of the linear expression given by Eq.(55) instead of the, only marginally more exact Eq.(3), is especially advantageous because allows for a trivial averaging over a finite energy range. The averaging of a complex energy-dependent expression is traded off by the averaging of a single parameter which depends linearly on the energy. An small solar neutrino contribution is still expected at $\cos \theta \sim 0.5$. We have explicitly checked that the results to be presented below, which use the available information about the slope sign, are independent of the concrete angle range used for fitting, in practice we have found rather stable results when we fit any number of bins up cosine values in the range $\cos \theta<0.25-0.5$.

\begin{tabular}{|c|c|c|c|}
\hline \hline Live Time & $(P\langle\alpha\rangle)_{\exp }$ & $P<P_{0}$ & $\Phi_{\bar{\nu}}\left({ }^{8} B\right)$ \\
\hline 504 days & $1.6 \times 10^{-3}$ & 0.075 & $<3.4 \times 10^{5}$ \\
700 days & $8.8 \times 10^{-4}$ & 0.040 & $<2.1 \times 10^{5}$ \\
825 days & $7.7 \times 10^{-4}$ & 0.035 & $<1.8 \times 10^{5}$ \\
\hline \hline
\end{tabular}

TABLE II. $(P\langle\alpha\rangle)_{\text {exp }}$ : slope fit to the experimental angle distributions $\left(E_{t h}>6.5 \mathrm{MeV}\right)$, CI95\% (unified Feldman-Cousins Approach with the boundary condition $\left.(P\langle\alpha\rangle)_{E x p}>0\right)$. Limits on the antineutrino probability supposing $\left\langle\alpha_{\text {eff }}\right\rangle=0.022$ (see text). Bounds on the antineutrino flux $\Phi_{\bar{\nu}}\left({ }^{8} B\right)\left(\mathrm{cm}^{-2} \mathrm{~s}^{-1}\right)$ (see Table(II)).

In order to draw a limit corresponding to a visible energy bin of width $\Delta E_{i}$, the fitted slope $b$ must be compared with the expected value $\alpha_{\exp }=Q_{0} / M\left\langle\alpha_{e f f}^{(1)}\right\rangle_{\Delta E_{i}}$, where $\left\langle\alpha_{e f f}^{(1)}\right\rangle_{\Delta E_{i}}$ is the energy average of Eq.(6) over the energy range $\Delta E_{i}$ and $M$ is the number of bins in which the full angle range is partitioned. From Eqs.(3) and (6), we obtain

$$
<\alpha_{e f f}^{(1)}>_{\Delta E_{i}}=-0.092+2.55 \times 10^{-3}\left\langle E_{e^{+}}\right\rangle_{\Delta E_{i}} .
$$

For example, for the full range $6.5-20 \mathrm{MeV},\left\langle E_{e^{+}}\right\rangle \simeq 10.0$ $\mathrm{MeV}$ and the value for the observable distribution param- 
eter $\left\langle\alpha_{e f f}^{(1)}\right\rangle \simeq-0.022$. Note that this value is far from the naive value for $\alpha^{0}=-0.1$ quoted above. Note in addition that detector angular resolution effects have been ignored here, according to estimations (see MC simulations above) they would induce a further, modest, smearing out of about $10-15 \%$ which conservatively has been included in the results. At higher energies and for small bin widths the recoil corrections are important and have throughly been included.

The results obtained from the fit to the full energy range distributions are shown in Table (II). An important improvement with respect to the bounds derived from the global background is obtained. The model independent upper limit on the ${ }^{8} B$ antineutrino flux is now $\Phi_{\bar{\nu}}\left({ }^{8} \mathrm{~B}\right)<1.8 \times 10^{5} \mathrm{~cm}^{-2} \mathrm{~s}^{-1}$, which corresponds to an upper bound $P<3.5 \%$ CI95. In order to extract CI intervals, we have used the known information about the sign of the expected slope: the unified Feldman-Cousins approach for dealing with boundary problems 19] has been used for this purpose.

A similar analysis was performed for each of the individual energy bins and for the cumulated distributions. The results are shown in Figs.(2) (bottom figure, empty circles) and (3) (empty circles). We see from the figures the complementary nature of both type of analysis: sensible limits are obtained only for energies down $10 \mathrm{MeV}$, the region where the bounds obtained from consideration of the global background are weaker. One draws a combined limit of $P<8 \%$ CI95\% for the full energy range. The bounds are in fact lower than the $5 \%$ level for the great part of the interval.

In summary, there are no indications of electron antineutrinos in the up-to-date SK data. This negative result sets upper bounds on the total and differential solar antineutrino spectrum. We obtain an upper bound $P_{\nu \rightarrow \bar{\nu}}<\approx 3.5 \%$ CI95\% above the $6.5 \mathrm{MeV}$ threshold. As a function of the energy, the stricter limits $(\sim 2 \%)$ are obtained for the highest positron visible energies.

This work has been supported by DGICYT under Grant PB95-1077 and by a DGICYT-MEC contract at Univ. de Valencia.

[1] E. Torrente-Lujan, Phys. Lett. B441 (1998) 305-312.

[2] E. Torrente-Lujan, Phys. Rev. D59, 093006 (1999).

[3] E. Torrente-Lujan, Phys. Rev. D59, 073001 (1999).

[4] V.B. Semikoz, E. Torrente-Lujan, Nucl. Phys. B 556 (1999) 353-372.

[5] F. Reines, R. M. Woods, Phys. Rev. Lett. 14 (1965) 20.

[6] G. Fiorentini, M. Moretti, F.L. Villante. hep-ph/9801111.

[7] K. Inoue, Talk at 8th Int. Workshop on Neutrino Telescopes, Venice, 23-18 February 1999.
[8] J.N. Bahcall, P.I. Krastev and Y. Smirnov, Phys. Rev. D58, 096016 (1998)

[9] P. Vogel, J.F. Beacom, hep-ph/9903554.

[10] G. Zacek et al. Phys. Rev. D34,9 (1986)2621.

[11] D. H. Wilkinson, Nucl. Phys. A377, 474 (1982).

[12] C. Caso et al., Eur. Phys. J. C 3, 1 (1998).

[13] H. Ishino. Ph.d Thesis. U. of Tokio (1999)

[14] M. Nakahata et Al (SK Collaboration) Nucl. Inst. Methods 46(1998)301.

[15] E. Torrente-Lujan, In preparation.

[16] M.B. Smy. hep-ex/9903034.

[17] M. Nakahata. Talk presented at TAUP99, Paris, Sep. 7, 1999.

[18] J.N. Bahcall and M.H. Pinsonneault, Rev. Mod. Phys. 67 (1995) 781.

[19] G.J. Feldman, R.D. Cousins, Phys. Rev. D57, 3873 (1998). 